\title{
CLOSING STABLE AND UNSTABLE MANIFOLDS ON THE TWO SPHERE
}

\author{
CLARK ROBINSON ${ }^{1}$
}

\begin{abstract}
Let $f$ be a diffeomorphism of the two sphere. In this note we prove that if the unstable manifold of a fixed point $p$ for $f$ accumulates on the stable manifold of $p$, then $f$ can be approximated arbitrarily closely $C^{r}, r \geqq 1$, such that they intersect.
\end{abstract}

1. The results. Let $d$ be a distance on the two sphere $S^{2}$ coming from a Riemannian metric. The stable manifold of a point $p$ is defined to be

$$
W^{s}(p, f)=\left\{x \in S^{2}: d\left(f^{n} x, f^{n} p\right) \rightarrow 0 \text { as } n \rightarrow \infty\right\} .
$$

The unstable manifold of $p$ is the stable manifold for $f^{-1}, W^{u}(p, f)=$ $W^{s}\left(p, f^{-1}\right)$. Let $W^{s}(p, f)-\{p\}=W^{s}(p, f)^{\prime}$ and $W^{u}(p, f)-\{p\}=W^{u}(p, f)^{\prime}$. A fixed point $p$ is called a saddle point if the eigenvalues $\lambda, \mu$ of the derivative $D f(p)$ satisfy $0<|\lambda|<1<|\mu|$.

THEOREM. Let $f$ be a $C^{r}$ diffeomorphism of $S^{2}, r \geqq 1$, and $p$ a fixed saddle point of $f$ such that $W^{s}(p, f)^{\prime} \cap$ closure $W^{u}(p, f) \neq \varnothing$. Then $f$ can be approximated arbitrarily closely $C^{r}$ by $f^{\prime}$ such that $f^{\prime}(p)=p$ and $W^{s}\left(p, f^{\prime}\right)^{\prime} \cap$ $W^{u}\left(p, f^{\prime}\right) \neq \varnothing$.

COROLLARY. There is a residual subset (complement of a first category set) $R$ of the set of all $C^{r}$ diffeomorphisms, $\operatorname{Diff}^{r}\left(S^{2}\right)$, such that if $f \in R, p$ is a saddle fixed point of $f$, and $W^{s}(p, f)^{\prime} \cap$ closure $W^{u}(p, f) \neq \varnothing$ then $W^{s}(p, f)^{\prime} \cap$ $W^{u}(p, f) \neq \varnothing$.

The reason for restricting to $S^{2}$ is to use the Jordan separation theorem. Therefore analogous results are true on the two disk and $R^{2}$. In the case of the disk, the above theorem should prove useful to prove a conjecture of Smale in [6]. For this use it would be nice to prove the result assuming only that $\{p\} \neq$ closure $W^{s}(p, f) \cap$ closure $W^{u}(p, f)$.

Next, I would hope the results would be true for periodic points but was unable to prove it. There are all sorts of related closing lemma conjectures. See [5].

Received by the editors February 24, 1973.

AMS (MOS) subject classifications (1970). Primary 58F10.

${ }^{1}$ This research was partially supported by the Organization of American States and the National Science Foundation, GP 19815.

c) American Mathematical Society 1973 
The only real examples I know of that satisfy the hypothesis of the theorems are cycles. The theorem was proved for these by Palls in [3]. I used this type of thinking to develop the present proof.

I would like to thank S. Newhouse who introduced me to this problem many years ago.

2. Proof of theorem. Assume $W^{s}(p, f)^{\prime} \cap$ closure $W^{u}(p, f) \neq \varnothing$ but $W^{s}(p, f)^{\prime} \cap W^{u}(p, f)=\varnothing$. (Otherwise we are done.) Let

$$
W_{\varepsilon}^{s}(p)=\left\{x \in W^{s}(p, f): d\left(f^{n} x, p\right) \leqq \varepsilon \text { for } n \geqq 0\right\} .
$$

This is the local stable manifold. Similarly,

$$
W_{\varepsilon}^{\prime \prime}(p)=\left\{x \in W^{u}(p, f): d\left(f^{n} x, p\right) \leqq \varepsilon \text { for } n \leqq 0\right\} .
$$

$W^{s}(p, f)^{\prime}$, closure $W^{u}(p, f)$, and $W^{s}(p, f)^{\prime} \cap$ closure $W^{u}(p, f)$ are invariant under $f$ so $W_{\varepsilon}^{s}(p, f)^{\prime}$ nclosure $W^{u}(p, f) \neq \varnothing$ for every $\varepsilon>0$.

Let the eigenvalues of $D f(p)$ be $\mu, \lambda$ where $0<|\lambda|<1<|\mu|$. Assume $D f(p)$ preserves the orientation of both the stable and unstable direction so $0<\lambda<1<\mu$. We make the modifications for the other cases below. Take $y \in W_{\varepsilon}^{s}(p, f)^{\prime} \cap$ closure $W^{u}(p, f)$. We want to construct a fundamental neighborhood of $y$. Let $T$ be a transversal to $W_{\varepsilon}^{s}(p, f)$ such that $y$ lies between $T \cap W_{\varepsilon}^{s}(p, f)$ and $f(T) \cap W_{\varepsilon}^{s}(p, f)$. Connect the two ends of $T$ to the two ends of $f(T)$ to enclose a region $Q$ such that $y \in$ interior $Q$, $Q \cap f(Q)=f(T)$, and $f^{j}(y) \notin Q$ for $j \geqq 1$.

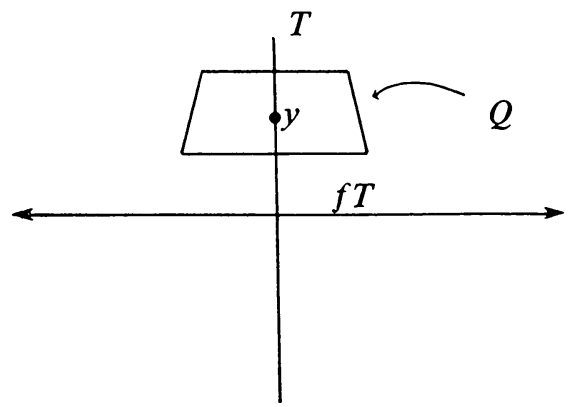

Such a region can easily be constructed in local coordinates that linearize $f$ near $p$ given by Hartman's theorem.

Let $x_{n}$ be the point of $f^{n} W_{1}^{u}(p, f)$ that is closest to $y$. Since

$$
\bigcup\left\{f^{n} W_{1}^{u}(p, f): n \geqq 0\right\}=W^{u}(p, f)
$$

we have that $x_{n}$ converges to $y$. Let $L_{0}=W_{\delta}^{s}(p, f)$ be such that $L_{0} \cap T$ 
is one point. Let $L=f L_{0}$. Let $N$ be an integer such that, for $n \geqq N, d\left(y, x_{n}\right)<$ $d(y, \partial Q)$ and $d\left(y, x_{n}\right)<d(y, L)$. Let $\mathcal{O}_{-}(z)=\left\{f^{-j}(z): 0 \leqq j\right\}$ be the backward orbit of $z$. For $n \geqq N, x_{n} \in \mathcal{O}_{-}\left(x_{n}\right) \cap$ interior $Q$.

LEMMA. For $n \geqq N, x_{n}=\mathcal{O}_{-}\left(x_{n}\right)$ ninterior $Q$.

Proof. For $z \in W^{u}(p, f)$ let $[p ; z]$ be the part of $W^{u}(p, f)$ between $p$ and $z$ including both end points. Assume $q=f^{-j}\left(x_{n}\right) \in$ interior $Q$ for $j \geqq 1$. Let $Q^{\prime}$ be the component of $Q-L_{0}$ such that $q \in Q^{\prime}$. Let $r$ be the first point as we move from $p$ to $q$ along $[p, q]$ such that $r \in Q^{\prime}$. We want to show $f^{j}[p, r]$ contains a point closer to $y$ than $f^{j}(q)=x_{n} \cdot f^{j}[p, r] \subset$ $f^{j}[p, q]=\left[p, x_{n}\right] \subset f^{n} W_{1}^{u}(p, f)$ so this contradicts definition of $x_{n}$. Therefore it contradicts $f^{-j}(q) \in$ interior $Q$ and proves the lemma.

Let $S_{0}$ be the part of the boundary of $Q^{\prime}$ such that $P=S_{0} \cup L_{0} \cup[p, r]$ is a simple closed curve. Let $D_{0}$ be the disk bounded by $P$ such that $Q^{\prime} \subset D_{0}$. Here we use Jordan separation theorem on the two sphere. $f Q^{\prime} \subset D_{0}$ because $\partial D_{0}$ does not cut across $f Q^{\prime}$ (by choice of $r$ ). Let $D_{1}=f D_{0}$ and $D_{k}=\bigcup\left\{f^{i}\left(D_{1}\right): 0 \leqq i \leqq k-1\right\} . y \notin D_{1}$. Let $S=f S_{0} . \partial D_{1}=S \cup L \cup[p, f r]$. The reader can check that for $k \geqq 2$

$$
\partial D_{k}-S-L \subset f\left(\partial D_{k-1}-S-L\right)
$$

and hence by induction

$$
\partial D_{j}-S-L \subset f^{j-1}\left(\partial D_{1}-S-L\right) \subset f^{j-1}[p, f r]=f^{j}[p, r]
$$

or

$$
\partial D_{j} \subset S \cup L \cup f^{j}[p, r]
$$

This boundary never crosses $L_{0}-L$ so $L_{0}-L$ all lies on the same side of $\partial D_{j}$. This is the opposite side from $f\left(Q^{\prime}\right) \subset D_{j}$. Therefore $y \in L_{0}-L$ is not in $D_{j}$. The point of $D_{j}$ closest to $y$ must lie on $\partial D_{j} . x_{n}=f^{j}(q) \in$ interior $D_{j}$ is closer to $y$ than $S$ and $L$ so the closest point is on $f^{j}[p, r]$. This is closer to $y$ than $x_{n}$. This completes the proof of the lemma.

ADDED IN PROOF. It is not necessary that $D_{j}$ be a disk for $j \geqq 2$. However, $L_{0}-L$ lies in one component of $S^{2}-\partial D_{j}$. This is a different component than $f\left(Q^{\prime}\right) \subset D_{j}$.

Thus we have constructed a sequence $x_{n} \in$ interior $Q, n \geqq N$, that converges to $y$, and $\mathcal{O}_{-}\left(x_{n}\right) \cap$ interior $Q=x_{n}$. Also $\mathcal{O}_{+}(y) \cap$ interior $Q=y$.

Now assume we are given $\varepsilon>0$ and want to approximate $f C^{r}$ within $\varepsilon$. By a standard argument, see [1], there exists $\delta>0$ such that if $z \in Q$ and $d(y, z)<\delta$, then there exists a function $f^{\prime}$ within $\varepsilon$ of $f$ in the $C^{r}$ topology such that $f^{\prime}(z)=f(y)$ and $f(x)=f^{\prime}(x)$ for $x \notin Q$. Take $n \geqq N$ large enough 
such that $d\left(y, x_{n}\right)<\delta$. Take $f^{\prime}$ as above. $p \notin Q$ so $f^{\prime}(p)=f(p)=p$ is a fixed point. $f^{\prime}\left(x_{n}\right)=f(y)$. By induction, $\left(f^{\prime}\right)^{k}\left(x_{n}\right)=f^{k}(y) \notin Q$. Therefore $\left(f^{\prime}\right)^{k}\left(x_{n}\right)$ converges to $p$ as $j$ goes to infinity. Therefore $x_{n} \in W^{s}\left(p, f^{\prime}\right) . f^{-1}(x)=$ $\left(f^{\prime}\right)^{-1}(x)$ for $x \notin f(Q)$. By induction $\left(f^{\prime}\right)^{-k}\left(x_{n}\right)=f^{-k}\left(x_{n}\right) \notin f(Q)$. Therefore $\left(f^{\prime}\right)^{-k}\left(x_{n}\right)$ converges to $p$ and $x_{n} \in W^{u}\left(p, f^{\prime}\right)$. This completes the proof in the case that $f$ preserves orientation.

If either eigenvalue of $f$ is negative we need to modify the proof. Let $g=f^{2}$. Take a transversal $T$ and use $T$ and $g(T)$ to construct the neighborhood $Q$ of $y$. $Q_{0}=Q-f(Q)$. Then $f^{j}(y) \notin Q_{0}$ for $j \geqq 1$. Let $L_{0}$ be as before and $L=g L_{0}$. Take $N$ such that $d\left(x_{n}, y\right)<d(y, \partial Q), d\left(y, \partial Q_{0}\right), d(y, L)$.

In the lemma assume $q=f^{-j}\left(x_{n}\right) \in$ interior $Q_{0} . \quad x_{n} \in Q_{0}$ and local analysis about $p$ shows $j \geqq 1$. Let $q, r, S_{0}, D_{0}$ be as before. Let

$$
\begin{gathered}
D_{2}=g D_{0}, \quad D_{2 k}=\bigcup\left\{g^{i}\left(D_{2}\right): 0 \leqq i \leqq k-1\right\}, \\
D_{2 k+1}=f D_{2 k}, \quad \text { and } S=g S_{0} . \\
\partial D_{2}=S \cup L \cup[p, f r] . \\
\partial D_{2 k} \subset S \cup L \cup f^{2 k}[p, r], \\
\partial D_{2 k+1} \subset f(S) \cup f(L) \cup f^{2 k+1}[p, r] .
\end{gathered}
$$

$x_{n} \in$ interior $D_{j}$ so we get a contradiction as before to prove $\mathcal{O}_{-}\left(x_{n}\right) \cap$ interior $Q_{0}=x_{n}$.

When we construct $f^{\prime}$ we require $f(x)=f^{\prime}(x)$ for $x \notin Q_{0}$ and $f^{\prime}\left(x_{n}\right)=$ $f(y) . \mathcal{O}_{+}(y) \cap Q_{0}=y$ and $\mathcal{O}_{-}\left(x_{n}\right) \cap Q_{0}=x_{n}$ so as before $x_{n} \in W^{s}\left(p, f^{\prime}\right) \cap$ $W^{u}\left(p, f^{\prime}\right)$.

3. Proof of corollary. We construct a semicontinuous function and use the method of Pugh [4] to prove the corollary.

Let $P(f)=\left\{p \in S^{2}: p\right.$ is a saddle fixed point of $f$ and $W^{s}(p, f)^{\prime}$ and $W^{u}(p, f)$ intersect transversally $\}$. Let $F$ be the family of closed subsets of $S^{2}$ with the Hausdorff metric. $P: \operatorname{Diff}^{r}\left(S^{2}\right) \rightarrow F$. Let $\operatorname{Diff}^{r}\left(S^{2}\right)$ have the usual uniform $C^{r}$ topology. By the persistence of transverse intersection [1], $P$ is lower semicontinuous. The points at which $P$ is continuous forms a residual subset $R \subset \operatorname{Diff}^{r}\left(S^{2}\right)$, see [2].

We claim $R$ satisfies the corollary. Assume not and there exist $f \in R$, and $p$ a saddle fixed point of $f$ such that $W^{s}(p, f)^{\prime} \cap$ closure $W^{u}(p, f) \neq \varnothing$ and $W^{s}(p, f)^{\prime} \cap W^{u}(p, f)=\varnothing$. Let $N$ be any $C^{r}$ neighborhood of $f$. By the theorem we can approximate $f$ by $f^{\prime} \in N$ such that $p$ is a saddle fixed point of $f^{\prime}$ and $W^{s}\left(p, f^{\prime}\right)^{\prime} \cap W^{u}\left(p, f^{\prime}\right) \neq \varnothing$. Then we can approximate $f^{\prime}$ by $f^{\prime \prime} \in N$ such that this intersection is transverse. Therefore $p \in P\left(f^{\prime \prime}\right)$. Since $N$ is an arbitrary neighborhood of $f$ and $P$ is continuous at $f$ we get $p \in P(f)$. Contradiction. 


\section{REFERENCES}

1. R. Abraham and J. Robbin, Transversal mappings and flows, Benjamin, New York, 1967. MR 39 \#2181.

2. G. Choquet, Lectures on analysis. Vol. I, Integration and topological vector spaces, Benjamin, New York, 1969. MR 40 \#3252.

3. J. Palls, A note on $\Omega$-stability, Proc. Sympos. Pure Math., vol. 14, Amer. Math. Soc., Providence, R.I., 1970, pp. 221-222. MR 42 \#5276.

4. C. Pugh, An improved closing lemma and a general density theorem, Amer. J. Math. 89 (1967), 1010-1021. MR 37 \#2257.

5. M. Shub, Stability and genericity for diffeomorphisms, 1971 Brazilian Sympos. on Dynamical Systems, M. Peixoto (editor), Academic Press (to appear).

6. M. Shub and S. Smale, Beyond hyperbolicity, 1971 Brazilian Sympos. on Dynamical Systems, M. Peixoto (editor), Academic Press (to appear).

Department of Mathematics, Northwestern University, Evanston, Illinois 60201 